\title{
Welche Praxis nach der postkolonialen Kritik? Human- und physisch-geographische Feldforschung aus übersetzungstheoretischer Perspektive
}

\author{
S. Husseini de Araújo ${ }^{1}$ and P. Kersting ${ }^{2}$ \\ ${ }^{1}$ Friedrich-Alexander-Universität Erlangen-Nürnberg, Institut für Geographie, \\ Kochstr. 4/4, 91054 Erlangen, Germany \\ ${ }^{2}$ Johannes Gutenberg-Universität Mainz, Geographisches Institut, \\ Johann-Joachim-Becher-Weg 21, 55099 Mainz, Germany \\ Correspondence to: S. Husseini de Araújo (shusseini@geographie.uni-erlangen.de) and \\ P. Kersting (p.kersting@geo.uni-mainz.de)
}

\begin{abstract}
Zusammenfassung. After the postcolonial critique, dealing with the power of speaking and self-reflexivity belong to the great challenges of academic work. In this article, we derive the necessity to accept these challenges from our own projects and discuss their practical consequences as well as the difficulties of integrating them in development studies of human and physical geography. We argue that the propositions of postcolonial theory cannot be transferred in practice without contradictions. Therefore, we try to grasp these contradictions with the concept of translation. From the point of view of Translation Studies, contradictions do not necessarily lead to failure, but they have the potential to create new knowledge and give voice to new perspectives.
\end{abstract}

\section{Geographische Entwicklungsforschung, postkolo- niale Kritik und Forschungspraxis}

Nicht ohne rhetorischen Hintergedanken fragt der Geograph Fabrice Ripoll „Peut-on ne pas être postcolonial?... surtout quand on est géographe?“ (2006), denn die postkoloniale Kritik erscheint viel zu schwerwiegend und zu weitreichend, als dass sich die Geographie ihr gegenüber verschließen könnte. Da die Geographische Entwicklungsforschung und die Postcolonial Studies bereits aufgrund ihrer Forschungsgegenstände zahlreiche Berührungspunkte aufweisen, verwundert gerade der geringe Austausch zwischen diesen beiden Forschungsrichtungen (McEwan, 2009:1; Radcliffe, 2005; Simon, 2006). Arbeiten, die sich in ihrem Schnittfeld verorten lassen, beschäftigen sich überwiegend mit selbstreflexiven Analysen und fragen nach den postkolonialen Machtverhältnissen und Epistemologien, die den „eigenen“ Forschungsprojekten und Lehrveranstaltungen im oder über „den Globalen Süden“ unterliegen (Abbot, 2006; Kapoor, 2004; McEwan, 2009:295f; Raghuram und Madge, 2006). Auffällig ist, dass gegenüber den dekonstruktivistischen Herangehensweisen dieser Arbeiten kaum empirische Studien im Bereich der Geographischen Entwicklungsfor- schung vorliegen, die auf Ansätzen der postkolonialen Theorie basieren und darauf abzielen, ,positives Wissen“ zu produzieren. Ist hier der Abstand zwischen Theorie und Praxis vielleicht einfach zu groß?

Mit unserem Beitrag wollen wir dieser Frage auf den Grund gehen. Anknüpfend an unsere Erfahrungen aus human- und physisch-geographischen Forschungsprojekten legen wir im Folgenden die Notwendigkeit einer Auseinandersetzung mit unserer Positionalität und Sprachmacht sowie einer kritischen Selbstreflexivität dar. Darauf aufbauend diskutieren wir die jeweiligen Annahmen der postkolonialen Theorie im Hinblick auf ihre praktische Umsetzung. Hierbei ergibt sich eine Reihe von Widersprüchen, die eine Vereinbarkeit der theoretischen Annahmen und Forderungen mit den Rahmenbedingungen der empirischen Arbeit schwierig bzw. unmöglich machen. Auf der Suche nach einer Lösung für dieses Problem greifen wir im abschließenden Schritt auf das Konzept der Übersetzung zurück und zeigen, wie dies als Scharnier zwischen postkolonialer Theorie und Praxis der Entwicklungsforschung fungieren kann, um solche Widersprüche nicht als ein Scheitern zu verstehen, sondern als eine Chance nutzen zu können. 


\section{Für Andere sprechen}

Eduardo da Mata (Name verändert) ärgert sich über die Nichtregierungsorganisationen (NRO) im Vale do Ribeira. Es handelt sich um seine Heimatregion, die als ökologisch reichste und zugleich als ökonomisch ärmste Gegend des Bundesstaates São Paulo in Brasilien gilt. Die NROs, so Eduardo, hätten hier zu Beginn eine sehr gute Arbeit geleistet. Inzwischen aber hätten sie alles monopolisiert. Dabei könnte seine Gemeinde selbst die Bananen vermarkten und sich um ein Öko-Siegel bemühen, wenn die Handlungsmacht nicht nur bei den NROs liegen würde (da Mata, Gespr. v. 17.3.2011).

Im Interview veranschaulichte Eduardo besonders eindrücklich, was es bedeutet, wenn Privilegierte für "Andere“ sprechen, wenn sie zu wissen glauben, was gut und richtig für ,die Anderen“ ist, während „den Anderen“ keine eigene Stimme und damit keine eigenen Entscheidungs- und Handlungskompetenzen ,zugesprochen“ werden. Problematisch ist dabei nicht nur die Bevormundung selbst, sondern auch die Gefahr, bestehende Sprach- und Machtverhältnisse zu stabilisieren (vgl. dazu Alcoff, 1992:7). So befürchtet auch Eduardo, dass viele der NROs im Vale do Ribeira die bestehenden gesellschaftlichen Strukturen eher verfestigen als aufbrechen, insbesondere wenn sie sich selbst als dauerhafte Einrichtungen verstehen. Obwohl ihr Anliegen der Ausgleich sozialer und ökonomischer Ungleichheiten sei, würden sie, um sich selbst und ihre Arbeit zu legitimieren, einen wirklichen Wandel in diese Richtung unterbinden (da Mata, Gespr. v. 17.3.2011).

Solche und ähnliche Beispiele finden sich zahlreich, und zwar nicht nur in der Entwicklungszusammenarbeit, in kolonialen Regierungs- oder in postkolonialen Interventionspolitiken (Easterly, 2006). Vom Problem, für Andere zu sprechen, ist auch die Wissenschaft maßgeblich betroffen (Said, 2009). Als Geograph_innen sind wir im Rahmen unserer Forschungsarbeiten im „Globalen Süden“ häufig die Sprachmächtigeren, die einen Umgang mit dieser Position finden müssen - gleich, ob wir Handlungsempfehlungen für die Entwicklungspolitik erarbeiten, wissenschaftliche Gutachten verfassen, Forschung betreiben oder Exkursionen durchführen. Vor diesem Hintergrund wäre auch nachvollziehbar, wenn Eduardo neben der Legitimität der NROs im Vale do Ribeira auch meine Legitimität als Wissenschaftlerin (Husseini de Araújo) und die meines Forschungsprojektes, das sich noch in der Konzeptionsphase befindet, infrage stellt.

\subsection{Forschen über, forschen mit oder nicht mehr forschen?}

Für die Suche nach forschungspraktischen Antworten auf die von Eduardo aufgeworfene Legitimitätsfrage bieten die Postcolonial und Postdevelopment Studies auf theoretischer Ebene anregende Denkanstöße. Gemeinsam ist ihnen eine ab- lehnende Haltung gegenüber den Praktiken, für „Andere“ im „Globalen Süden“ zu sprechen, zu wissen und zu entscheiden, da diese vor allem dazu führten, (post-)koloniale Strukturen zu reproduzieren (Alcoff, 1992). In der Konsequenz werden diese Praktiken einerseits selbst zum Forschungsgegenstand. Ziel dabei ist, die Machtverhältnisse offenzulegen, die dem Sprechen über und für „Andere“ unterliegen, und zu zeigen, inwieweit dieses Sprechen jene Machtverhältnisse fortschreibt. Andererseits wird nach einer Forschungspraxis gesucht, die auf eine „,angemessene“ Weise mit dem Problem, für „Andere“ zu sprechen, umgeht. Doch was als ,angemessen" gilt, wird äußerst kontrovers diskutiert.

Die Positionen reichen von einer radikalen Abkehr von Forschung im bzw. über den „Globalen Süden“ bis hin zu Forderungen nach Forschungskooperationen (letztere findet sich beispielsweise auch im Förderungsinstrument der Deutschen Forschungsgemeinschaft (DFG) „Kooperation mit Entwicklungsländern“ wieder). Vertreter_innen der postkolonialen Kritik verknüpfen diese Forderung nach Forschungskooperationen oftmals auch mit der Notwendigkeit, kontinuierliche Interventionsmöglichkeiten durch die Beforschten zu gewährleisten und einen Wissensrückfluss sicherzustellen (ebd., McEwan, 2009). Dabei sollte die Entwicklungsforschung im Idealfall ,die Sichtweise der Betroffenen im Süden auf gesellschaftliche Probleme und Zielsetzungen zwar nicht immer unkritisch übernehmen, aber zunächst einmal zum Ausgangspunkt machen, um so die Definitionsmacht von den Experten auf die Subalternen zu übertragen“ (Ziai, 2006:215).

\subsection{Probleme auf dem Weg zu einem gleichwertigen Sprechen}

Doch sei es das Plädoyer für die Verabschiedung von Forschung im „Globalen Süden“ oder der versöhnlichere Appell, „die Sichtweise der Betroffenen im Süden [...] zum Ausgangspunkt zu machen" (ebd.) und Partnerschaften im Sinne eines „Forschen[s] mit statt über“ (Müller-Mahn und Verne, 2010:7) zu initiieren: In beiden Fällen erweisen sich die theoretisch plausiblen Forderungen auf forschungspraktischer Ebene als kaum einlösbar.

Die Ausgangssituation im Vale do Ribeira zeigt bereits, dass Denkkategorien, die durch die postkoloniale Theorie zur Verfügung stehen, viel zu undifferenziert sind und in Teilen auch nicht sinnvoll erscheinen. So lässt sich nicht nur schwer beantworten, wo der „Globale Süden“ anfängt und aufhört, sondern auch die Frage, wer oder was „die Anderen“, „die Subalternen“ oder „die Betroffenen im Süden“ sind, deren Sichtweise zum Ausgangspunkt gemacht werden soll. Diese Kategorien haben zunächst einmal keinen Platz für brasilianische Wissenschaftler_innen, Machthaber_innen, bürokratische Apparate oder politische Aktivist_innen „vor Ort“, genauso wenig für die NROs im Vale do Ribeira, über die Eduardo sich ärgert, oder für Eduardo selbst. Vielmehr stellt sich die Frage, ob er nicht entsetzt wäre, wenn er oder die 
Mitglieder seiner Gemeinde von „uns“ als „subaltern“ oder „Andere“ bezeichnet würden. Anders ausgedrückt: Reproduzieren wir durch Kategorien wie „Betroffene im Süden“ und „Subalterne“ als „die Anderen“ und wir als „die Entwicklungsforscher_innen“ nicht genau jene gesellschaftlichen Differenzierungen und Essentialisierungen eines modernistischen und kolonialen Denkens, die wir zu überwinden suchen (Lossau, 2002)?

Darüber hinaus ist unklar, wie ein gleichberechtigtes „Forschen mit" angesichts der meist asymmetrischen Verteilung materieller und immaterieller Kapitalien gestaltet werden könnte. Dieses Problem kann sich durch Universitätsund Forschungsförderungsstrukturen, die mit „unseren“ auf bürokratischer Ebene nicht kompatibel sind - und dies gilt in weiten Teilen auch für die brasilianischen -, noch verstärken. $\mathrm{Zu}$ befürchten wäre daher, dass die Konzeptionsmacht aufgrund bestehender institutioneller Strukturen und Verfügungsgewalten über finanzielle Mittel vornehmlich in ,unseren“ Händen bleibt (beispielsweise im Rahmen eines DFG-Projektes, das durch das Instrument „Kooperation mit Entwicklungsländern“ gefördert wird). Dann könnte eine gleichzeitige Einforderung von Partizipation dazu führen, dass unser Kooperations- und Partnerschaftsgedanke in einem Drang zur Kooptierung post-demokratischer Art mündet (Korf, 2010). Diese Gefahr ist ein grundsätzliches Problem, was in vielen Forschungskontexten des globalen Südens beobachtet wird. So spricht der beninische Philosoph Paulin J. Hountondji (1990:7) von einer ,epistemischen Gewalt des Nordens“ und beklagt die „Extrovertiertheit“ sowie ,die wissenschaftliche Abhängigkeit Afrikas“ (vgl. dazu auch Foaleng, 2003 oder Macamo, 2010).

\section{Selbstreflexivität}

Eng verflochten mit dem Problem, für „Andere“ zu sprechen, ist die Selbstreflexivität. Dass sich die Frage des Umgangs mit diesen beiden Aspekten nicht nur im Bereich der Human-, sondern auch in der Physischen Geographie stellt, soll im Folgenden anhand eines abgeschlossenen Forschungsprojektes über die Landschaftsgenese des ruandischen zentralen Hügellandes veranschaulicht werden (Kersting, 2010a). Zwei Impulse waren hier ausschlaggebend: Zum einen zeigte sich ein deutlicher Widerspruch zwischen den ,europäischen“ Beschreibungen dramatischer Erosionsprozesse in Ruanda und der weitaus weniger besorgten ,ruandischen" Einschätzung derselben. Dieser Unterschied hat schwerwiegende Konsequenzen, denn während im ersten Fall eine „Entwicklungszusammenarbeit" dringend notwendig erscheint, relativiert sich die Notwendigkeit im zweiten Fall. Zum anderen wurde ich (Kersting) aufgrund meiner deutsch-französischen Staatsbürgerschaft immer wieder mit der Rolle Frankreichs während des Genozids von 1994 konfrontiert. Um den Genozid verstehen zu können, ist die Kenntnis der Geschichte der Geschichte der ruandischen
Ethnien eine wesentliche Voraussetzung. Das hierfür zugrunde liegende Konzept der Ethnogenese ermöglicht, die ruandischen Ethnien als performative Konstrukte zu begreifen, die aus wechselseitigen Fremd- und Eigenzuschreibungen entstehen (Neubert und Brandstetter, 1996). Ausgehend von diesem Impuls stellte sich im Rahmen des Forschungsprojektes die Frage, inwieweit diese konstruktivistische und performative Bedeutung des Suffixes ,-genese“ ebenfalls in den Begriffen Morphogenese und Landschaftsgenese mitgedacht werden kann bzw. muss.

Die Beantwortung dieser Frage erfordert eine Betrachtung der eigenen physisch-geographischen Wahrnehmung. Dabei kann der zentrale morphogenetische Prozess der Bodenerosion nicht mehr als ein natürlicher, objektiver und quantifizierbarer Prozess angenommen werden, der ,für sich spricht". Die Methodik, die der Messung und Quantifizierung von Bodenabtragsraten zugrunde liegt, muss als das Ergebnis sozialer Aushandlungsprozesse betrachtet werden (Osbahr und Allan, 2003; Warren et al., 2001). So besitzt bereits die Feststellung des Phänomens der Bodenerosion eine gesellschaftliche Dimension (Rossi, 1997). Sichtbar wurde diese im Kontext des Forschungsprojektes beispielsweise darin, dass die ruandischen Mitarbeiter_innen die auf den Projektfeldern propagierten Erosionsschutztechniken auf ihren eigenen Feldern nicht anwendeten.

Vor diesem Hintergrund müssen die Bedeutung und Wirkmächtigkeit der Begriffe, Konzepte und Theorien, mit denen Geomorpholog_innen und Agrarökolog_innen über Bodenerosion in Ruanda sprechen, ins Blickfeld genommen werden. Was bedeutet der Begriff Erosion im ruandischen Kontext? Woher kommen die damit assoziierten Vorstellungen von Stabilität und Labilität? Wie wird die wechselseitige Beziehung zwischen Erosion und Gesellschaft gedacht? Seit wann stellt die europäische Wissenschaft die Frage der Bodenerosion in Ruanda? Warum kündigen europäische Wissenschaftler_innen seit nun fast einem Jahrhundert in regelmäßigen Abständen die sehr baldige endgültige Zerstörung der ruandischen Böden an? Wie, wann und warum haben sich Beschreibung und Bewertung des Prozesses gewandelt? Warum werden auf den Erosionsmessparzellen so hohe Abtragungswerte gemessen bzw. wie werden diese Werte (un-)bewusst ,hergestellt“? Worauf deutet die Tatsache, dass die ruandische Übersetzung des Begriffs „Erosion“ eher dem Konzept der Denudation entspricht (Kersting, 2010b; Krings, 2002; Rossi, 1997)? Angesichts dessen, dass die Erosionsforschung zu tief greifenden landschaftlichen und gesellschaftlichen Veränderungen geführt haben und weiterhin führen - vgl. u.a. die Zwangsarbeit, die Einrichtung von rund $300.000 \mathrm{~km}$ Hecken und Erosionsschutzgräben in Ruanda (König, 1992:64) sowie die derzeitige Landpolitik -, darf die performative Wirkung dieser Theorien, Konzepte und Begriffe nicht außer Acht gelassen werden. 


\subsection{Dekonstruktion als Ziel der Selbstreflexivität. .}

Auch hinsichtlich der Frage der Selbstreflexivität bietet die postkoloniale Kritik Anknüpfungspunkte, die weit über eine bloße Ablehnung des Objektivitätsgedankens und eine Forderung nach Positionierung hinausgehen. $\mathrm{Zu}$ den Schlüsselwerken gehören in diesem Zusammenhang insbesondere die Schriften Gayatri C. Spivaks (1988, 1990). In seinem Beitrag „Hyper-self-reflexive development? Spivak on representing the Third World ,Other" "“ arbeitet der Politikwissenschaftler Ilan Kapoor fünf Schritte aus ihren Studien heraus, die als mögliche Grundlage selbstreflexiver Entwicklungsforschung dienen können (Kapoor, 2004:640ff.). Hinter dem ersten Schritt ,„Intimately inhabiting ' and ,negotiating discourse" verbirgt sich die Annahme, dass Dekonstruktion und Kritik eines Diskurses nur innerhalb desselben erfolgen können. Den Entwicklungsdiskurs könnten wir demnach nicht von außen kritisieren, sondern müssten anerkennen, dass wir im Moment der Kritik zum Teil desselben werden. So wäre beispielsweise auch die Untersuchung der Bodenerosion in Ruanda als Teil des europäischen Entwicklungsdiskurses zu betrachten, den es von dieser Position heraus neu auszuhandeln gilt. Beim zweiten Schritt geht es um die Anerkennung von Komplexität, da wir, so die Annahme, als Subjekte in einer Vielzahl unterschiedlicher Diskurse situiert sind. Die beiden folgenden Schritte ,Unlearning one's privilege as loss“" und „Learning to learn from below“ werden als notwendig erachtet, um ,den Anderen“ als Subjekt und nicht als Objekt von Entwicklung zu betrachten. Das „Verlernen“ soll vor der eigentlichen Feldforschung erfolgen und erfordert zunächst eine Zurückverfolgung der Geschichte unserer Vorurteile und gelernten Gewohnheiten, von Rassismus, Klassendenken und Sexismus bis hin zum akademischen Elitendasein und Ethnozentrismus. Dies soll uns von den „eigenen“ Wissens- und Repräsentationssystemen befreien und ermöglichen, vom „Anderen“ zu lernen. Im Falle des Bodenerosionsprojektes in Ruanda würde dies nicht nur bedeuten, die eigenen Wahrnehmungs- und Deutungskategorien zu erkennen und zu hinterfragen, sondern sie komplett abzulegen. Was in den Vordergrund rückt, sind die Erklärung, Deutung und Bewertung der Bodenerosionsprozesse durch ruandische Kolleg_innen, ruandische Kleinbäuerinnen und Kleinbauern sowie die Wissens- und Repräsentationssysteme, in denen deren Erklärungen, Deutungen und Bewertungen eingebunden sind. Eng mit diesem Schritt des Lernens vom „Anderen“ verbunden ist der letzte: die Bereitschaft, ohne Erfolgsgarantien zu arbeiten. Es muss möglich werden, auch Scheitern als ein Ergebnis zu akzeptieren und als Erfolg deuten zu können.

\section{$3.2 \ldots$ oder Selbstreflexivität als Ausgangspunkt für Konstruktion?}

Innerhalb der postkolonialen Theorie wurden Spivaks Ansätze zur Selbstreflexion in vielerlei Hinsicht kritisiert. Vorgeworfen wird ihr insbesondere, in ihren Ausführungen oftmals unvollständig und unklar zu bleiben (Kapoor, 2004). Dieser Kritikpunkt zeigt sich noch einmal deutlicher auf forschungspraktischer Ebene, da sich ein großer Teil der Vorschläge, trotz einer nachvollziehbaren Argumentation, als kaum umsetzbar erweist. Wie können wir „ohne Garantien“ arbeiten, wenn lediglich erfolgsversprechende Forschungsarbeiten gefördert, zugelassen und erfolgreiche Forschung belohnt wird? Das Bodenerosionsprojekt war in eine größere Forschungskooperation zwischen einer ruandischen und einer deutschen Universität eingebunden, die u.a. vom Ministerium des Inneren und für Sport des Landes RheinlandPfalz finanziell unterstützt wurde. Vor einem solchen Hintergrund lässt sich weder einfordern, ohne Garantien arbeiten zu können, noch vor der Feldforschung einen Prozess des „Verlernens“ einzuleiten. Im Gegenteil: Erst nach gründlicher Vorbereitung und einem Prozess des Lernens wurden Forschende ,ins Feld gelassen“.

Darüber hinaus stellt sich die Frage, wie ein „Verlernen“ vor der eigentlichen Feldforschung erfolgen soll, wenn wir die hegemonialen Diskurse, in die wir verstrickt sind, nie in Gänze überblicken können, wie z. B. die Reichweite des Diskurses über Labilität und Stabilität von Umwelt und Gesellschaft in den Tropen (vgl. Kersting, 2010a)? Und was tun, wenn unsere Forschungspartner_innen im „Globalen Süden“ alles andere als das „Verlernen“ unserer Repräsentations- und Wissenssysteme sowie eine Dekonstruktion unseres Tuns erwarten? Nur allzu oft wurden in uns genau die Wissenschaftler_innen gesehen, die wir vor dem Hintergrund der postkolonialen Kritik überwinden wollten. Als problematisch erscheint in diesem Zusammenhang auch die rein dekonstruktivistische Herangehensweise, denn sie lässt keinen Spielraum für die Produktion von ,,positivem“ Wissen und birgt die Gefahr, in der Selbstreflexion, bestenfalls beim Lernen von „Anderen“", stecken zu bleiben. Aus einer Perspektive der geographischen Entwicklungsforschung kann Dekonstruktion nicht das Ziel sein, sondern nur ein erster Schritt, auf den ein zweiter folgt, mit dem ,positives Wissen“ produziert werden darf.

\section{Geographische Entwicklungsforschung und post- koloniale Kritik: Überlegungen aus übersetzungs- theoretischer Perspektive}

Die Auseinandersetzungen mit der Sprachmacht sowie die Selbstreflexivität im Sinne der postkolonialen Kritik erscheinen uns - nicht zuletzt aufgrund unserer eigenen Erfahrungen - für geographische Arbeiten unerlässlich, insbesondere auch für die geographische Entwicklungsforschung. Gleichwohl haben unsere bisherigen Ausführungen beispielhaft gezeigt, dass der Versuch kaum gelingen kann, die theoretisch formulierte Kritik auf forschungspraktischer Ebene konsequent umzusetzen. Trotz dieses Dilemmas lehnen wir den Appell einiger Vertreter_innen der postkolonialen Theorie, gar nicht im oder über den „Globalen Süden“ zu forschen, genauso ab wie eine Abkehr der Entwicklungsforschung von 
der postkolonialen Theorie. Wir sind vielmehr der Ansicht, dass eine Entwicklungsforschung, die versucht, die postkoloniale Kritik mitzudenken und nicht nur dekonstruktivistisch $\mathrm{zu}$ arbeiten, trotz aller Schwierigkeiten ein fruchtbares Unterfangen sein kann. Als konzeptionelle Grundlage für ein solches Unterfangen möchten wir die Denkfigur der Übersetzung vorschlagen, da sie die Widersprüche und Schwierigkeiten zu greifen vermag, die sich im Zusammenhang mit den forschungspraktischen Konsequenzen aus den theoretischen Forderungen der postkolonialen Kritik ergeben. Übersetzung ist selbst ein großes Themenfeld der postkolonialen Theorie, wird in diesem Kontext jedoch eher auf kulturelle und kulturimperialistische Übersetzungsprozesse bezogen (z.B. Spivak, 2009; Bhabha, 1990). Wir setzen hier einen anderen Fokus und rücken die Forschungspraxis ins Zentrum.

\section{1 Übersetzung als Praxis dazwischen}

Ausgangspunkt unserer Überlegungen ist, dass wir nie $a u$ thentisch für „Andere“ sprechen können, sondern die Stimmen „der Anderen“ in den Diskurs unseres Wissenschaftskontextes übersetzen (Husseini de Araújo, 2011:116ff.; Husseini, 2009). Vom linguistischen Paradigma ausgehend dürfen die Übersetzungen nicht als Eins-zu-Eins-Übertragungen von Bedeutung verstanden werden. Es handelt sich um hybride Stimmen, die aus den unvermeidbaren Bedeutungsverschiebungen hervorgehen. Diese Vorstellung fußt auf der Annahme, dass Original, Übersetzende (Forschende) und übersetzte Texte (Forschungsergebnisse) stets auf spezifische und letztlich einzigartige Weisen kontextualisiert sind (Toury, 1982). Entscheidende Größen eines Wissenschaftskontextes sind beispielsweise die Wissenschaftsdiskurse, kulturelle und ideologische Repräsentationssysteme, persönliche Zusammenhänge, Zwecke und Ziele der Forschung sowie Verständnis, Wissen, Ansprüche und Fähigkeiten der Forschenden. Dieser Kontext unterliegt zwar ständigen Veränderungen, nicht zuletzt durch die Übersetzungsschritte selbst (Iser, 1994), doch bleibt der Blick auf „den Anderen“ immer im ,eigenen“ Kontext gefangen (Frow, 1995). Daher ist auch ein „Über“-Setzen zum „Anderen“ nicht möglich, sondern immer nur ein Dazwischen. Diese Position eröffnet einerseits einen neuen Verhandlungsraum von Repräsentation und Bedeutung, der ein produktives Moment beinhaltet und eine Brücke zwischen „Eigenem“ und „Anderem“ baut. Andererseits birgt dieses Dazwischen auch etwas Zerstörerisches in sich, da ein „Über“-Setzen nie gelingen kann und die Übersetzung immer eine Veränderung des Originals bedeutet (Venuti, 2003). Diese Ambivalenz der Übersetzerrolle manifestiert sich nur allzu deutlich im italienischen Sprichwort „Traduttore, traditore!“ (,Übersetzer_in, Verräter_in!“", Üb. d. V.).

\section{2 „Forschen dazwischen“ anstelle von „Forschen mit statt über"}

Analog zu diesem Verständnis von Übersetzung ist eine Umsetzung der theoretischen Forderungen der postkolonialen Kritik in die Praxis der geographischen Entwicklungsforschung auch nicht eins-zu-eins möglich, sondern immer mit Bedeutungsverschiebungen verbunden. Dabei können wir nicht ohne Essentialisierungen, Exotisierungen, Domestizierungen oder Hierarchisierungen auskommen, denn wir bewegen uns als Forschende und Übersetzende nicht in einem strukturellen Niemandsland; der forschende Blick bleibt stets im eigenen Kontext gefangen. Aus diesem Grund müssen wir auch von bestehenden Machtverhältnissen erst einmal ausgehen, selbst wenn diese asymmetrisch sind und wir ein Forschen ,auf Augenhöhe“ mit unseren Kooperationspartner_innen im „Globalen Süden“ bevorzugen würden. Gleichwohl ist der Kontext in vielen Teilen auch flexibel und viele Rahmenbedingungen können bereits durch einzelne Übersetzungsschritte verschoben werden - dies gilt für einige der Rahmenbedingungen mehr, für andere, wie beispielsweise finanzielle, weniger.

Aus übersetzungstheoretischer Perspektive dürfen asymmetrische Machtverhältnisse daher nicht verschwiegen, sondern müssen mitgedacht und ausbuchstabiert werden. Bereits dies kann helfen, sie zu verändern (wenn teilweise auch nur geringfügig). Als Beispiel lässt sich aus dem Forschungsprojekt in Ruanda anführen, dass die ruandischen Wissenschaftler_innen, Kleinbäuerinnen und Kleinbauern seit über 100 Jahren unzählige Erosionsforschungsprojekte aus dem „Globalen Norden“ gesehen haben. Die Forscher_innen aus Europa ignorierten und ignorieren dabei häufig die lange Beforschungsgeschichte der Beforschten. Für sie ist ihr Forschungsprojekt meist ein einmaliges Ereignis, für die ruandische Seite ist es lediglich ein weiteres Projekt in einer langen Kette der exogenen Be- bzw. Erforschung. Würde diese Beforschungsgeschichte mitgedacht und anerkannt, könnte sich dadurch nicht nur die Kommunikationsbasis mit den ruandischen Kolleg_innen (als eine der Rahmenbedingungen) verändern, sondern dies vielleicht auch zum stärkeren Bemühen um ein Verständnis darüber beitragen, warum die ruandischen Mitarbeiter_innen auf den Projekt- und Demonstrationsfeldern die propagierten Anbaumethoden und auf den eigenen Felder ihre eigenen Techniken anwenden (vgl. dazu Kersting, 2010a:5ff.).

Was Kategorien und theoretische Konzepte wie „Subalterne“, „Betroffene im Süden“ und „Entwicklungsforscher_innen" betrifft - oder wie im Falle des Projekte in Ruanda: „exzessive Tiefenerosion“, „Erosion“ und „Entwicklung“ ", so mögen diese vielleicht zu Beginn des Forschungsprozesses als angemessen oder einzig vorhandenen erscheinen, mit denen sich arbeiten lässt, auch wenn sie bis zu einem gewissen Grad modernistische oder gar eurozentristische Denkstrukturen reproduzieren. Aus übersetzungstheoretischer Sicht geht es nun nicht darum, solche 
Kategorien und Konzepte vor Beginn der Feldforschung zu verlernen, so wie seitens der postkolonialen Kritik gefordert wird, denn ein solches Verlernen erscheint aufgrund der Kontextgebundenheit und Pfadabhängigkeit von Forschung ohnehin nicht möglich. Aufgabe ist vielmehr, die Kategorien und Konzepte im Laufe des Forschungs- und Übersetzungsprozesses durch das Lernen vom „Anderen“ zu verändern, neu $\mathrm{zu}$ justieren, andere und passendere Konzepte zu finden. Dies gilt bereits für die Begriffe, mit denen wir „uns“ und „unsere Anderen“ definieren. Sowie aus den „Subalternen“ oder „Betroffenen im Süden“ „Quilombolas im Vale do Ribeira“ oder „ruandische Kleinbäuerinnen und Kleinbauern“ werden, lässt sich beispielsweise ebenso auf die Kategorie „Entwicklungsforscher_innen“ verzichten. Das Projekt in Ruanda zeigte ferner, dass auch theoretische Konzeptualisierungen von beobachteten Phänomenen durch die Übersetzungsschritte im Forschungsprozess verändert werden können. Die Tatsache, dass im Kinyarwanda beispielsweise von ,die Steine wachsen“ die Rede ist, deutet darauf hin, dass der beobachtete Prozess als flächenhaft (Denudation) und nicht als linienhaft (Erosion) wahrgenommen wird (die differenzielle Abtragung führt zum flächenhaften $\mathrm{Ab}$ transport des feinkörnigen Materials, so dass der Eindruck entsteht, die gröberen, nicht mobilisierten Fraktionen (Steine) würden aus der Fläche ,herauswachsen“). Diese Erkenntnis hilft, die tiefgreifenden Unterschiede in den ,ruandischen“ und ,europäischen“ Bewertungen des Bedrohungspotentials besser nachzuvollziehen, zwischen den unterschiedlichen Perspektiven und Auffassungen (Denudation vs. Erosion) zu vermitteln und die eigenen Konzeptualisierungen zu modifizieren.

Ein solches Vermitteln zwischen Eigenem und Anderem, zwischen Theorie und Praxis ist ein „Dazwischen“, das aufgrund der unvermeidlichen Bedeutungsverschiebungen zumeist keiner Seite von jeweils beiden wirklich gerecht werden kann, aber dennoch Brücken baut. Vor diesem Hintergrund möchten wir für die Metapher vom „Forschen dazwischen“ eintreten, die die Umsetzungsproblematik von Forderungen der postkolonialen Kritik in die Praxis der Geographischen Entwicklungsforschung nicht verschweigt, wie die Metapher vom „Forschen mit statt über“, sondern mitberücksichtigt. Dieses Dazwischen verstehen wir als ein Charakteristikum von Übersetzenden, das ihnen ,eine strukturelle Selbstentfremdung auf[erlegt], die an Schizophrenie erinnert" (Flusser, 2004, in Guldin, 2005:17). Wer sich auf eine solche Schizophrenie einlassen kann, ist unserer Ansicht nach auch in der Lage, die postkoloniale Kritik mit der praxisbezogenen Geographischen Entwicklungsforschung in einen konstruktiven Austausch zu bringen - einen Austausch, der ermöglicht, aus gegenseitiger Kritik zu lernen, neues, auch ,positives Wissen“ zu produzieren und damit die Kluft zwischen beiden Forschungsrichtungen zu überwinden.

\section{Literatur}

Abbot, D.: Disrupting the 'whiteness' of fieldwork in geography, Singapore J. Trop. Geo., 27, 326-341, 2006.

Alcoff, L.: The Problem of Speaking for Others, in: Cultural Critique 1991-92, 5-32, 1992.

Bhabha, H.: The Third Space. Interview with Homi Bhabha, in: Identity. Community, Culture, Difference, Herausgeber: Rutherford, J., London, 207-221, 1990.

Da Mata, E. (Name verändert): Aktivist, Quilombo de Ivaporunduva (persönliche Kommunikation vom 17.3.2011), 2011.

Easterly, W.: The White Man's Burden. Why the West's Efforts to Aid the Rest Have Done So Much Ill and So Little Good, New York, The Penguin Press, 2006.

Foaleng, M.: Wissenschaftskooperation und Rassismus - Hindernisse in der Zusammenarbeit zwischen deutschen und afrikanischen Wissenschaftlern, Entwicklingspolitik, 5, 33-36, 2003.

Frow, J.: Cultural Studies and Cultural Value, Oxford, Oxford University Press, 1995.

Guldin, R.: Philosophieren zwischen den Sprachen, Vilém Flussers Werk, München, Wilhelm Fink Verlag, 2005.

Hountondji, P.: Scientific Dependence in Africa Today, Res. Afr. Literatures, 21/3, 5-15, 1990.

Husseini de Araújo, S.: Jenseits vom ,Kampf der Kulturen“. Imaginativer Geographien des Eigenen und des Anderem in arabischen Printmedien, Bielefeld, Transcript, 2011.

Husseini, S.: Die Macht der Übersetzung - Konzeptionelle Überlegungen zur Übersetzung als politische Praktik am Beispiel kulturgeographischer Forschung im arabischen Sprachraum, Soc. Geogr., 4, 71-81, doi:10.5194/sg-4-71-2009, 2009.

Iser, W.: On Translatability, Surfaces, 4, 5-13, 1994.

Kapoor, I.: Hyper-self-reflexive development? Spivak on representing the Third World "Other", Third World Q., 25/4, 627-647, 2004.

Kersting, P.: Geomorphologische Untersuchungen im Land der tausend Hügel - oder: wie europäisch ist die ruandische Landschaftsentwicklung?, Forum IfL 13, Leipzig, 1-194, 2010a.

Kersting, P.: Où sont les paysages rwandais? Paysagenèse: un regard postcolonial, in: trajectoires - travaux des jeunes chercheurs du ciera 4 - http://trajectoires.revues.org/index493.html (last access: 1 March 2011), 2010b.

König, D.: Erosionsschutz in Agroforstsystemen. Möglichkeiten zur Begrenzung der Bodenerosion in der kleinbäuerlichen Landwirtschaft Rwandas im Rahmen standortgerechter Landnutzungssysteme, Mainz, Selbstverlag des Geographischen Instituts Mainz (= Mainzer Geographische Studien, 37), 1992.

Korf, B.: The geography of participation, Third World Q., 31/5, 709-720, 2010.

Krings, T.: Zur Kritik des Sahel-Syndromansatzes aus Sicht der Politischen Ökologie, Geogr. Z., 90/3+4, 129-141, 2002.

Lossau, J.: Die Politik der Verortung. Eine postkoloniale Reise zu einer ANDEREN Geographie der Welt, Bielefeld, Transcript, 2002.

Macamo, E.: Entwicklungsforschung und Praxis. Kritische Anmerkungen aus der Sicht eines Beforschten, Geographische Rundschau 10, 52-57, 2010.

McEwan, C.: Postcolonialism and Developement, New York u.a., Routledge, 2009. 
Müller-Mahn, D. und Verne, J.: Geographische Entwicklungsforschung - alte Probleme, neue Perspektive, Geographische Rundschau, 10, 4-11, 2010.

Neubert, D. und Brandstetter, A.-M.: Einleitung: Historische und gesellschaftliche Hintergründe des Konflikts in Ruanda, in: Staat und Gesellschaft in Afrika: Erosions- und Reformprozesse, Herausgeber: Meyns, P., Hamburg, LIT. (= Schriften der VAD, 16), 409-424, 1996.

Osbahr, H. und Allan, C.: Indigenous views of soil erosion at Fandou Béri, southwestern Niger, Geoderma, 111/3-4, 439-456, 2003.

Radclife, S.: Development and geography: towards a postcolonial development geography?, Progess in Human Geography, 29/3, 291-298, 2005.

Raghuram, P. und Madge, C.: Towards a method for postcolonial development geography? Possibilities and challenges, Singapore J. Trop. Geo., 27/3, 270-288, 2006.

Ripoll, F.: Peut-on ne pas être postcolonial?... surtout quand on est géographe, in: EspacesTemps.net - http://espacestemps.net/ document2136.html (last access: 1 March 2011), 2006.

Rossi, G.: Notre érosion et celle des autres, Cahiers d'Outre-Mer, 50, 57-68, 1997.
Said, E.: Orientalismus, Frankfurt, Fischer, 2009.

Simon, D.: Separated by common ground? Bringing (post)development and (post)colonialism together, The Geographical Journal, 172/1, 10-21, 2006.

Spivak, G. C.: Can The Subaltern Speak?, in: Marxism and the Interpretation of Culture, edited by: Nelson, C. and Grossberg, L., Urbana, Chicago, 271-313, 1988.

Spivak, G. C.: Gayatri Spivak on the politics of the subaltern. Interview by Howard Winant, Socialist Rev., 20/3, 81-97, 1990.

Spivak, G. C.: The Politics of Translation, in: The Translation Studies Reader, edited by: Venuti, L., New York u.a., 369-388, 2009.

Toury, G.: A Rationale for Descriptive Translation Studies, Dispositio, 7, 22-39, 1982.

Venuti, L.: The Scandals of Translation. Towards an Ethics of Difference, London u.a., Routledge, 2003.

Warren, A., Batterbury, S., und Osbahr, H.: Soil erosion in the West African Sahel. A review and an aplication of a "local political ecology" approach in South West Niger, Global Environ. Chang., 11, 79-95, 2001.

Ziai, A.: Post-Development. Ideologiekritik in der Entwicklungstheorie, Politische Vierteljahresschrift, 47/2, 193-218, 2006. 